\title{
Concordancia del diagnóstico del cirujano con el diagnóstico histopatológico en pacientes adultos intervenidos quirúrgicamente por apendicitis aguda
}

\author{
Concordance of the diagnosis of the surgeon with histopathological diagnosis in adult \\ patients surgical intervention by acute appendicitis
}

\author{
Gamaliel Vázquez-Estudillo ${ }^{1 *}$, Edwin Y. Ochoa-Viveros ${ }^{1}$, Ivanhoe Larracilla-Salazar ${ }^{1,2}$, \\ Gerardo Rodarte-Cajica ${ }^{2}$ y Murata Chiharu ${ }^{3}$ \\ ${ }^{1}$ Especialidad de Cirugía General, Escuela de Posgrados en Sanidad Naval; ${ }^{2}$ Servicio de Cirugía General, Hospital General Naval de Alta \\ Especialidad; ${ }^{3}$ Especialidad de Metodología, Escuela de Posgrados en Sanidad Naval. Ciudad de México, México
}

\begin{abstract}
Resumen
Objetivo: Identificar la concordancia del diagnóstico macroscópico con el diagnóstico histopatológico en pacientes intervenidos quirúrgicamente por apendicitis aguda (AA) en el Hospital General Naval de Alta Especialidad (HOSGENAES). Método: De los pacientes con probable AA operados de apendicetomía en el servicio de cirugía general del HOSGENAES se recuperó el reporte histopatológico con apoyo del archivo electrónico HIS-2 del hospital. La concordancia se realizó por el índice kappa de Cohen. Resultados: En este estudio de 200 casos de AA, al categorizar por grupos en relación a la fase de AA fueron diagnosticados por el cirujano 5 (2.5\%) casos como apéndices aparentemente sanos o sin signos inflamatorios, 73 (36.5\%) como AA congestiva-catarral, 97 (48.5\%) como flemonosa-supurada, 20 (10\%) como gangrenosa-necrosada y 5 (2.5\%) como perforada. El diagnóstico histopatológico arrojó las siguientes cifras: 10 (5\%) casos de apéndices sin signos inflamatorios, 61 (30.5\%) casos de AA congestiva o catarral, 107 (53.5\%) flemonosa o supurada, 11 (5.5\%) como gangrenosa o necrosada, y 11 (5.5\%) como perforada. Conclusiones: Se obtuvo una baja concordancia (kappa: 0.18) en el diagnóstico de $A A$ entre cirujanos y patólogos al clasificarla por fases.
\end{abstract}

PALABRAS CLAVE: Apendicitis. Apendicectomía. Concordancia.

\begin{abstract}
Objective: To identify the concordance of the macroscopic diagnosis with the histopathological diagnosis in patients surgicaIly treated for Acute Appendicitis (AA) in the Naval General Hospital of High Specialty (HOSGENAES). Methods: All the patients with probable $A A$ operated on appendectomy, in the general surgery service of the HOSGENAES, the histopathological report was recovered with support of the HIS-2 electronic file system of the hospital, the agreement was made by Cohen's kappa index. Results: In this study of 200 cases of $A A$, when categorized by groups in relation to the AA phase in the postoperative period, $5(2.5 \%)$ cases were diagnosed by the surgeon as appendages apparently healthy or without inflammatory signs, $73(36.5 \%)$ as congestive AA or catarrhal, 97 (48.5\%) as phlegmonous or suppurative, 20 (10\%) as gangrenous or necrotic and $5(2.5 \%)$ as perforated. Histopathological diagnosis yielded the following figures: $10(5 \%)$ cases of appendices without inflammatory signs, 61 (30.5\%) cases of congestive or catarrhal $A A, 107$ (53.5\%) phlegmonous or suppurative, 11 (5.5\%) as
\end{abstract}

\author{
Correspondencia: \\ *Gamaliel Vázquez-Estudillo \\ Calzada de la Virgen 203 int. 104 \\ Col. Culhuacán CTM, Del. Coyoacán \\ C.P. 04480, Ciudad de México, México \\ E-mail: dr.gama.mcn@gmail.com
}

Fecha de recepción: 04-04-2018

Fecha de aceptación: 05-06-2018

DOI: 10.24875/CIRU.18000317
Cir Cir. 2018:86:534-538

Contents available at PubMed www.cirugiaycirujanos.com 
gangrenous or necrotic and $11(5.5 \%)$ as perforated. Conclusions: We obtained a low concordance (kappa: 0.18) in the diagnosis of $A A$ among surgeons and pathologists when classifying it by phases.

KEY WORDS: Appendicitis. Appendectomy. Concordance.

\section{Introducción}

La apendicetomía es actualmente el procedimiento quirúrgico de urgencia más común en el mundo; el riesgo calculado de presentar apendicitis a lo largo de la vida es del $8.6 \%$ para los hombres y del $6.7 \%$ para las mujeres. Presenta una incidencia de 1.5 a 1.9 casos por cada 1000 habitantes, y la población mayormente afectada se encuentra entre los 15 y los 35 años de edad. Uno de cada 15-20 mexicanos presentará apendicitis aguda (AA) en algún momento de su vida, y en nuestro hospital se han realizado cerca de 800 apendicetomías en los últimos 5 años, lo que lleva a ser uno de los procedimientos quirúrgicos más frecuentes, al igual que en la literatura reportada'.

La AA es la patología quirúrgica de urgencia más frecuente en nuestro medio; sin embargo, continúa siendo un reto diagnóstico para los profesionales de la salud aun contando con todos los estudios de extensión y de mayor sensibilidad y especificidad'.

Se han propuesto y creado diversas escalas diagnosticas para AA, siendo la escala de Alvarado la más conocida y la más utilizada, ya que se ha validado su uso en numerosos estudios. Hasta la fecha, aún no ha sido identificado un marcador específico fiable de AA. A pesar de los avances en la tecnología y las modalidades de investigación, el porcentaje de apendicetomías negativas se mantiene entre el 15 y el $50 \%$. Los estudios muestran que existe cierto beneficio al realizar una tomografía en pacientes que presentan dolor abdominal con datos clínicos pocos sugestivos de AA, ya que los criterios utilizados en la escala de Alvarado la mayoría de las veces se presentan en fases tardías de apendicitis".

Es ampliamente conocido que la sospecha de apendicitis es clínica; se integra mediante anamnesis, exploración física, estudios de laboratorio y gabinete; y se corrobora mediante estudios histopatológicos, pues una vez que se realiza el procedimiento quirúrgico es estadificado por el cirujano de acuerdo con las fases macroscópicas (fase $0-\mathrm{IV}$ ), pero esta clasificación en ocasiones difiere de los reportes histopatológicos.

La fase de la apendicitis no se confirma hasta los hallazgos posoperatorios. De manera estandarizada, la fase determina los días de tratamiento farmacológico, los días de estancia hospitalaria y las posibles complicaciones; el diagnostico histopatológico definitivo puede modificar esta evolución. Consideramos prudente la búsqueda de concordancia en los diagnósticos, ya que se relaciona con el tratamiento adecuado, los días de estancia hospitalaria, los días de recuperación y las posibles complicaciones. La falla en realizar un diagnóstico temprano eleva la mortalidad y la morbilidad de la enfermedad, así como el riesgo de complicaciones. El diagnóstico retardado incrementa los costos en el servicio de urgencias y hospitalarios ${ }^{2,3}$.

La gravedad de la apendicitis puede determinarse por el aspecto macroscópico y por el examen histopatológico. Mediante la observación macroscópica de los hallazgos quirúrgicos se puede clasificar de la siguiente manera: fase 0 , sin apendicitis; fase I, apendicitis congestiva o catarral, en la cual se aprecia hiperemia; fase II, apendicitis flemonosa o supurativa, es decir, con erosiones de la mucosa, supuración y exudados fibrinopurulentos; fase III, apendicitis necrosada o gangrenosa de la pared; y fase IV, apendicitis perforada. Aunque hasta la fecha no existe un modelo único en cuanto a la clasificación clínica, en algunos hospitales es válida para la clasificación histopatológica ${ }^{4-7}$.

El manejo farmacológico va en relación con el estadio de la $A A$, y existen diversos tratamientos y esquemas. Los casos de AA no complicadas en fases I y II (congestivas y flegmonosas) requieren una estancia hospitalaria y un tratamiento antibiótico breves, en algunos casos 24 horas o menos; en lo que respecta al tratamiento de las apendicitis agudas complicadas en fases III y IV (gangrenadas y perforadas), estas requieren una estancia hospitalaria de 72 horas y un tratamiento antibiótico doble, de 7 a 10 días, que se inicia de manera intrahospitalaria y con manejo por vía oral para seguimiento externo $0^{8-12}$.

Conocer el porcentaje de casos en que se confirma el diagnóstico histopatológico de AA y su correlación con el diagnóstico macroscópico en nuestra institución ayudará en la aplicación del esquema de tratamiento antibiótico ideal, ya que este es dependiente de la fase de la $A A^{13-17}$. 
En la Secretaría de Marina, Armada de México, es prioridad mantener al personal militar en óptimas condiciones de salud para su desempeño en las diferentes actividades, y en el caso de presentar patologías de resolución quirúrgica, las metas que seguimos son disminuir la estancia intrahospitalaria, las complicaciones y la comorbilidad, tanto para militares en el activo como para retirados y derechohabientes; aquí radica la importancia de la adecuada clasificación macroscópica al realizar el procedimiento quirúrgico y la concordancia del diagnóstico definitivo, el cual sin lugar a dudas es el reporte histopatológico.

\section{Método}

Se llevó a cabo un estudio de tipo transversal cuantitativo no experimental, en el periodo comprendido de septiembre de 2015 a agosto de 2017, con todos los pacientes intervenidos quirúrgicamente con diagnóstico de $A A$ que acudieron a valoración médica y recibieron tratamiento quirúrgico en el Hospital General Naval de Alta Especialidad (HOSGENAES), los cuales incluyen personas de 16 a 65 años, siendo militares activos 0 retirados, derechohabientes y no derechohabientes.

Las variables a estudiar el fueron el sexo, la edad, las fases de apendicitis macroscópica y la clasificación histopatológica. La clasificación utilizada fue la siguiente: sin apendicitis, congestiva o catarral, flegmonosa o supurada, gangrenosa o necrosada, y perforada.

El tamaño de la muestra calculado fue de 193 pacientes ${ }^{18-20}$.Se estableció el nivel de confianza de un $95 \%$ con un margen de error de kappa \pm 0.15 .

Una vez que se aprobó el protocolo de investigación, se captaron los pacientes con apoyo del personal médico del servicio de cirugía general del HOSGENAES. Se recabó información del diagnóstico emitido por el cirujano en la nota posoperatoria y del diagnóstico histopatológico en los reportes del servicio de patología, con el apoyo del sistema de archivo electrónico HIS-2 del hospital.

El análisis de la concordancia entre los diagnósticos macroscópico e histopatológico se realizó mediante el índice kappa de Cohen.

\section{Resultados}

Este estudio de 200 casos de AA presentó una mayor proporción de pacientes del sexo femenino, con 114 contra 86 pacientes de sexo masculinos.
Tabla 1. Fases de apendicitis aguda según los diagnósticos macroscópico e hitopatológico en los pacientes sometidos a apendicectomía

\begin{tabular}{|c|c|c|c|c|c|c|c|}
\hline \multirow{3}{*}{ 음 } & & \multicolumn{6}{|c|}{ Diagnóstico histopatológico } \\
\hline & & 0 & 1 & 2 & 3 & 4 & Total \\
\hline & 0 & 2 & 3 & 0 & 0 & 0 & 5 \\
\hline \multirow{5}{*}{ 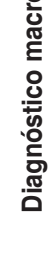 } & 1 & 7 & 26 & 39 & 1 & 0 & 73 \\
\hline & 2 & 1 & 32 & 60 & 3 & 1 & 97 \\
\hline & 3 & 0 & 0 & 8 & 6 & 6 & 20 \\
\hline & 4 & 0 & 0 & 0 & 1 & 4 & 5 \\
\hline & Total & 10 & 61 & 107 & 11 & 11 & 200 \\
\hline
\end{tabular}

La mayor incidencia de cuadros clínicos apendiculares se presentó en pacientes entre la tercera y la cuarta décadas de la vida, siendo mayoritaria la tercera década, con un total de 87 casos, con un rango de edad de 45 años entre el menor con 15 años y 60 años el paciente con mayor edad.

Al categorizar por grupos en relación a la fase de AA en el posoperatorio, fueron diagnosticados por el cirujano 5 (2.5\%) como apéndices aparentemente sanos o sin signos inflamatorios, $73(36.5 \%)$ como AA congestiva o catarral, 97 (48.5\%) como flemonosa o supurada, $20(10 \%)$ como gangrenosa o necrosada, y $5(2.5 \%)$ como perforada (Tabla 1). El diagnóstico histopatológico arrojó las siguientes cifras de resultados: $10(5 \%)$ apéndices sin signos inflamatorios, 61 (30.5\%) AA congestiva o catarral, 107 (53.5\%) flemonosa o supurada, 11 (5.5\%) gangrenosa o necrosada, y $11(5.5 \%)$ perforada (Tabla 1).

\section{Discusión}

El diagnóstico de AA mejora con el correr de los años de ejercicio del cirujano, por su experiencia y por los adelantos en medicina en estudios auxiliares, principalmente imagenológicos. A pesar de todo esto, no es infrecuente hallar un apéndice aparentemente sano durante la intervención quirúrgica en un paciente con diagnóstico presuntivo de AA. Tampoco es raro no encontrar una causa que justifique el dolor en la fosa iliaca derecha a la exploración del abdomen en estos pacientes ${ }^{3}$.

El porcentaje de apendicectomías negativas considerando el punto de vista del cirujano fue del $5 \%$, similar a lo citado por Kulikoff, et al. ${ }^{21}$ en su estudio de 782 casos pediátricos, ascendiendo al $10 \%$ cuando se comparó con el resultado de la anatomía patológica. Ambos estudios difieren de lo reportado por 
Ávila y García ${ }^{22}$ en Colombia, quienes presentan un $18.1 \%$ de apendicectomías negativas. Es importante destacar que el valor reportado en nuestro estudio se encuentra por debajo del $12-15 \%$ de laparotomías llamadas en blanco o negativas, que se considera tolerable desde el punto de vista quirúrgico según lo indicado por el comité de elaboración de guías de práctica clínica de la Asociación Mexicana de Cirugía General'. A pesar de esto, es necesario tener en cuenta que obtener menos del $12 \%$ de apendicectomías negativas también debe preocupar, ya que podría significar que pacientes con AA atípicas se dejen en observación y que sean operados cuando ya presenten alguna complicación.

Al categorizar las AA por fases se obtuvo una baja concordancia interobservador (kappa: 0,181085), similar a lo reportado por Segovia y Figueredo ${ }^{3}$ (kappa: $0,3466)$; sin embargo, difiere de los reportes de concordancia de Flores-Nava et al. ${ }^{17}$ en pacientes pediátricos, quienes presentan un $72.2 \%$ de similitud entre el reporte del cirujano y el del patólogo, lo cual contrasta con el $49 \%$ de similitud en nuestro estudio.

En general, los cirujanos y patólogos diagnostican más AA flegmonosas (48.5 y 53.5\%, respectivamente). También se encontró poca diferencia entre los diagnósticos macroscópico e histopatológico en las fases complicadas (12.5 y $11 \%$, respectivamente), con un $100 \%$ de efectividad al clasificar como AA complicada, similar al $96.7 \%$ reportado por Pourhabibi, et al. ${ }^{23}$ en 342 pacientes.

La falla en el diagnóstico de AA complicadas tiene importancia debido a la implicación en la terapéutica, los días de internamiento y la duración del tratamiento antibiótico. Los falsos negativos (53 casos, 26.5\%) que fueron subdiagnosticados, es decir, clasificados macroscópicamente en una fase menor en relación al reporte de histopatología, recibieron terapia antibiótica subóptima, lo que nos lleva a suponer que podría producirse una mayor tasa de morbilidad quirúrgica (más infecciones del sitio quirúrgico, abscesos u otras complicaciones) en muchos casos, aunque debería realizarse una evaluación más profunda para valorar esta situación, que no formó parte de este trabajo. Los falsos positivos (49 casos, $24.5 \%$ ) que fueron sobrediagnosticados tuvieron una estancia hospitalaria y un tratamiento antibiótico excesivos, que probablemente se deba al exceso de precaución con el objetivo de prevenir complicaciones posteriores.

En su momento, con pocos medios auxiliares de diagnóstico, McBurney ${ }^{24}$ propuso que una práctica quirúrgica rápida para asegurar la no progresión de la AA es mucho más segura que retardar el tratamiento quirúrgico, legado que persiste hasta la fecha bajo la frase «ante la duda, no hay duda", o sea, laparotomía; frase que se ha adoptado tanto en nuestro servicio de cirugía general como en muchos otros hospitales muy seguramente, lo que nos garantiza evitar en cierto modo procedimientos que impliquen mayor morbimortalidad para el paciente, el cual siempre es y será la prioridad para nosotros ${ }^{24,25}$.

\section{Conclusión}

Se obtuvo una baja concordancia (kappa: 0.18 ) en el diagnóstico de AA entre cirujanos y patólogos al clasificarla por fases. Teniendo en cuenta esto, lo ideal sería que hubiera una concordancia buena (kappa: > 0,7) o excelente (kappa: > 0,8) entre el diagnóstico macroscópico posoperatorio hecho por el cirujano y el histopatológico hecho por el patólogo de los casos de AA y sus fases o estadios, ya que la terapéutica posoperatoria depende del adecuado diagnóstico emitido por el cirujano al realizar el procedimiento quirúrgico.

\section{Recomendaciones}

- Que el cirujano sea más cuidadoso con los hallazgos macroscópicos, con el fin de afinar el diagnóstico posoperatorio.

- Emitir el reporte histopatológico en breve para realizar los ajustes en el tratamiento farmacológico intrahospitalario y extrahospitalario en caso de ser necesario.

- Que exista mayor comunicación entre los servicios de cirugía general y patología, y que en caso de existir duda se realice una sesión conjunta.

\section{Agradecimientos}

AI HOSGENAES por las facilidades aportadas para la elaboración de este trabajo de investigación.

\section{Conflicto de intereses}

Los autores declaran no tener ningún conflicto de intereses.

\section{Bibliografía}

1. Comité de elaboración de guías de práctica clínica. Guía de práctica clínica Apendicitis aguda. AMCG A.C. Ciudad de México; 2014. p. 1-25.

2. Ates MF, Khander A, Steigman SA, Tracy TF Jr., Luks FI. Use of white blood cell count and negative appendectomy rate. Pediatrics. 2014;133:e39-44. 
3. Segovia Lohse HA, Figueredo Thiel SJ. Surgical-pathologic concordance in the diagnosis of the acute appendicitis. An Fac Cienc Med. (Asunción). 2012;45:35-46.

4. Guzmán-Valdivia GG. Una clasificación útil en apendicitis aguda. Rev Gastroenterol Mex. 2003;68:261-5.

5. Bliss D, Mckee J, Cho D, Krishnaswami S, Zallen G, Harrison M, et al. Discordance of the pediatric surgeon's intraoperative assessment of pediatric appendicitis with the pathologists report. J Pediatr Surg. 2010;45:1398-403.

6. Yilmaz M, Akbulut S, Kutluturk K, Sahin N, Arabaci E, Ara C, et al. Unusual histopathological findings in appendectomy specimens from patients with suspected acute appendicitis. World J Gastroenterol. 2013;19:4015-22.

7. Ponsky T, Hafi M, Heiss K, Dinsmore J, Newman K, Gilbert J. Interobserver variation in the assessment of appendiceal perforation. J Laparoendosc Adv Surg Tech. 2009;19(Suppl 1):S15-8.

8. Rossem CC, Schreinemacher MH, Treskes K, van Hogezand RM, van Geloven AA. Duration of antibiotic treatment after appendicectomy for acute complicated appendicitis. Br J Surg. 2014;101:715-9.

9. Kimbrell A, Novosel T, Collins J, Weireter L, Terzian H, Beydoun $\mathrm{H}$, et al. Do postoperative antibiotics prevent abscess formation in complicated appendicitis? Am Surg. 2014;80:878-83.

10. Castañeda-Espinosa SD, Molina-Ramírez I, Holguín-Sanabria A, Jaimes-de La Hoz P, Perilla-López M, Pedraza-Carvajal A, et al. Cambio en la clasificación macroscópica de la apendicitis. ¿Tiene algún impacto? Estudio retrospectivo en un hospital universitario pediátrico. Rev Fac Med. 2015;63:243-50.

11. Priale PG, Mayta TP. Factors associated with negative appendectomy at a private hospital in Lima - Peru. IMedPub Journals. 2015;11:1-6.

12. Soto-López A, Águila-Melero O, Reyes-Corcho A, Consuegra-Díaz JE, Gómez-Baute R. Eficiencia diagnóstica en la apendicitis aguda. Cir Cir. 2003;71:204-9.

13. Suárez MO, Fausto $A$, Tapia Z. Interaprendizaje de estadística básica. Universidad técnica del norte. Ibarra, Ecuador; 2014(I). p. 15-6.

14. Liang M, Andersson R, Jaffe B. The appendix. Schwartz's Principles of surgery. $10^{\text {th }}$ ed. McGraw-Hill Education; 2014(30). p. 1241-62.
15. Macias-Magadan M, Cordero-Olivares A, Fonseca I. Apendicitis: incidencia y correlación clínico patológica. Experiencia de 5 años. Revista Mexicana de Cirugía Pediátrica. 2009;16:170-5.

16. Huacuja-Blanco RR, Ruiz-Campos M, Lemus-Ramírez RI, Villegas-Tovar E, González-Chávez MA, Díaz-Girón G, et al. Factores predictores para apéndice blanca y apendicitis aguda en pacientes sometidos a apendicectomía. Experiencia de dos años en una institución privada. Rev Invest Med Sur Mex. 2015;22:11-8.

17. Flores-Nava G, Jamaica-Balderas ML, Landa-García RA, Parraguirre-Martínez S, Lavalle-Villalobos A. Apendicitis en la etapa pediátrica: correlación clínico-patológica. Bol Med Hosp Infant Mex. 2005;62:196-201.

18. Cichetti DV. Testing the normal approximation and minimal sample size requirements of weighted kappa when the number of categories is large. Applied Psychological Measurement. 1981;5:101-4.

19. Donner A, Eliasziw M. Sample size requirements for reliability studies. Stat Med. 1987;6:441-8.

20. Fleiss JL. Comparison of the null distribution of weighted kappa and the C ordinal statistic. Applied Psychological Measurement. 1977;1:195-201.

21. Kulikoff $B$, Vargas $F$, Rodríguez $C$. Correlación clínico patológica en la apendicitis aguda pediátrica: revisión de 782 casos. Rev Ped Elec. 2009;6:121.

22. Ávila Mauricio J, García-Acero M. Apendicitis aguda: revisión de la presentación histopatológica en Boyacá, Colombia. Rev Colomb Cir. 2015;30:125-30.

23. Pourhabibi ZN, Javidi PP, Bolandparvaz S, Paydar S. Accuracy of surgeon's intraoperation diagnosis of acute appendicitis, compared with the histopathology results. Bull Emerg Trauma. 2014;2:15-21.

24. McBurney $\mathrm{C}$. The incision made in the abdominal wall in cases of appendicitis, with a description of a new method of operating. Ann Surg. 1894;20:38-43.

25. Martínez Mier G, Reyes Devesa HE. Mc Burney: the incision, the point, and the surgeon. Cirujano General. 2007;1:70-5 